\title{
Anoxia at Birth Induces Behaviorally-Relevant Changes in alpha2- Noradrenergic Receptor Binding in the Adult Rat
}

Wayne G. Brake ${ }^{* \underline{Y}}$, B.Sc., M.Sc., and $\underline{\text { Shakti Sharma }} \underline{\underline{Y}}$, B.Sc., M.Sc.

* To whom correspondence should be addressed: Ý Department of Neurology and Neurosurgery, Douglas Hospital Research Centre, McGill University, 6875 LaSalle Blvd., Verdun, QC, Canada H4H 1R3

\section{ABSTRACT}

Evidence suggests that Caesarean section birth in the rat, with or without an additional period of anoxia, results in long-term changes in brain catecholamine levels as well as reactivity to stress. The purpose of the present investigation was to determine whether Caesarean birth plus anoxia alters (alpha) $)_{2}$-noradrenergic receptor binding and sensitivity to the (alpha) $)_{2}$ receptor agonist, clonidine, in the Porsolt forced swim test. Sprague Dawley rat dams were decapitated and the uteruses were removed by Caesarean section. Pups were then delivered either immediately (Caesarean Only group), or were immersed in a saline bath for approximately 15 minutes (Caesarean plus Anoxia group) before delivery of the pups. A third group of animals born vaginally served as controls (Vaginally-born group). Four to five months postnatally, the expression of (alpha $)_{2}$ receptors was measured by receptor autoradiography using $\left[{ }^{3} \mathrm{H}\right]$-Rauwolscine binding. Receptor binding was increased in the area of the ventral hypothalamus and decreased in the $\mathrm{CA1}$ region of the hippocampus in animals subjected to Caesarean plus Anoxia at birth. These animals also displayed a subsensitive response to the immobilizing effects of clonidine (100 micrograms/kg, i.p.) in the Porsolt forced swim test. Specifically, these data show that Caesarean birth produces long-term changes in (alpha) $)_{2}$ receptor density and that, in animals subjected to Caesarean plus anoxia, these changes are reflected in a behavioral subsensitivity to the (alpha) ${ }_{2}$ agonist, clonidine. The findings reported here provide further experimental support for the hypothesis that birth complications may contribute to the pathophysiology of disorders such as schizophrenia that involve central catecholamine dysfunction.

\section{INTRODUCTION}

There is an emerging body of evidence correlating psychiatric disorders, schizophrenia in particular, to obstetric complications at the time of birth (1-10). While a number of such complications have been identified as potential risk factors for schizophrenia, including breached birth, delayed labor, Caesarean (C)section, or umbilical cord prolapse, the exact nature of the perinatal insult remains a matter of speculation. However, one consequence common to many of these perinatal complications is an episode of anoxia to the fetus. Exactly how intrauterine anoxia might increase the risk of developing schizophrenic symptoms is even less clear, but one possibility is by altering the function of brain catecholaminergic systems, particularly 
dopamine (DA) and norepinepherine (NE).

Dopamine has been implicated in the pathophysiology of schizophrenia, in part, from evidence that amphetamine (an indirect DA agonist) can elicit psychotic symptoms that are clinically indistinguishable from positive symptoms observed in paranoid schizophrenics (11-13). Furthermore, the clinical antipsychotic efficacy of the classical neuroleptics is directly related to their potencies in blocking $\mathrm{DA} \mathrm{D}_{2}$-like receptors (14-16). NE (also known as noradrenaline) is associated with schizophrenia because it has been shown to be elevated in postmortem brain and cerebrospinal fluid (CSF) of schizophrenics--especially in those suffering from positive symptoms or of the paranoid type (17-22).

Bjelke et al. reported evidence consistent with the idea that birth complications may affect catecholaminergic function (23). This study reported an increased number of tyrosine hydroxylase (TH)-immunoreactive cells in the ventral tegmental area (VTA) and substantia nigra of 21-day old rats that had been subjected to a period of intrauterine anoxia at birth. TH is an enzyme responsible for catalyzing the synthesis of catecholamines and thus serves as a marker for DA and NE neurons. Other studies have shown that perinatal anoxia induces long-term changes in various neurotransmitter systems, including dopamine (24), and short-term alterations in Fos immunoreactivity (25).

Previously, the present authors' group has found that an animal model of C-section birth, with or without an additional period of anoxia, can produce, upon adulthood, a sensitized nucleus accumbens DA response to repeated mild stress, resulting in enhanced stress-induced DA release (26). In addition, anoxia at birth facilitates sensitization to the locomotor stimulant effect of stress and amphetamine in adult rats (27). Taken together, these findings suggest that a period of anoxia to the fetus can lead to long-lasting changes in central DA function and increased sensitivity to the effects of repeated stress.

In the central nervous system, one of the principal components of the stress response is the locus coeruleus (LC) NE system, whose projections to the cortex, hippocampus and hypothalamic nuclei are instrumental in regulating the release of many hypothalamic peptides which drive the peripheral stress response (28). After a single exposure to any of a variety of stressors, intracellular NE levels are decreased, corresponding to an increase in activation-induced NE release from neurons (28). These NE neurons are regulated, in part, by inhibition through presynaptic (alpha) ${ }_{2}$-noradrenergic autoreceptors. Studies indicate that application of $*_{2}$ antagonists produce anxiety-like symptoms, presumably by increasing NE release.

The aim of the present investigation was to determine whether birth complications affect the function of NE systems in the adult brain. Specifically, the purpose was to determine whether birth by C-section or C-section with an additional period of transient anoxia may alter the density of brain (alpha) ${ }_{2}$-noradrenergic receptors and, if so, to determine whether such changes are behaviorally relevant.

\section{MATERIALS AND METHODS}

\section{Intrauterine anoxia}

All procedures involving animal subjects were carried out in strict accordance with the guidelines established by the Canadian Council on Animal Care. Intrauterine anoxia was induced using a modified version of the delayed C-section method described by Bjelke et al. (23). On the day of parturition, Sprague Dawley rat dams (Charles River, St. Constant, Canada) were decapitated and hysterectomized. The entire uterus, including the fetuses, was then removed and immediately immersed in a $37^{\circ} \mathrm{C}$ saline bath for approximately 15 minutes (Caesarean plus Anoxia); from the time of decapitation, it typically took 30-40 seconds to hysterectomize the dam and remove the uterus. The pups were then removed from the uterus and, if necessary, gently palpated to initiate breathing. Pups were placed on a heating pad until they were paired with a foster dam. In the present study, the period of anoxia was defined as the time the uterus was removed from the dam to the moment the 
pups began breathing on their own. The majority of pups began breathing normally within 60 seconds after removal from the uterus ${ }^{1}$.

The present study also included two groups of control animals. One group of controls comprised pups that were born vaginally (Vaginal Control). The other comprised animals that were removed from the uterus immediately after being delivered by C-section (Caesarean Only). Thus, apart from the 30-40 seconds during which the dam was hysterectomized and the uterus was removed and the 40-60 seconds required to initiate breathing in all the pups of the litter, animals in the Caesarean Only group were not subjected to any additional period of anoxia. Only male pups were included in the study to avoid the possibility of confounding group effects with sex differences. Pups were cross-fostered by surrogate dams; each dam was matched with an equal number of pups from each group. Upon weaning at 21 days, animals were dually housed on a $12 \mathrm{hr}$ light/dark schedule (lights on at 08:00 hours) with food and water available ad libitum.

\section{Receptor autoradiography}

At four months of age, three animals from each group were decapitated and the brains were immediately removed and frozen in isopentane $\left(-40^{\circ} \mathrm{C}\right)$ and then stored at $-80^{\circ} \mathrm{C}$. Frozen brains were sectioned at 15 micrometers thickness on the coronal plane, thaw-mounted onto gelatin-coated slides, desiccated under vacuum overnight at $4^{\circ} \mathrm{C}$, and then stored at $-80^{\circ} \mathrm{C}$.

For (alpha) ${ }_{2}$-noradrenergic receptor binding, coronal sections taken at the level of the striatum as well as the posterior hypothalamus were first preincubated for 10 minutes at room temperature in buffer containing 50 $\mathrm{mM}$ Tris- $\mathrm{HCl}, 10 \mathrm{mM} \mathrm{MgCl}_{2}, 4 \mathrm{mM} \mathrm{CaCl}_{2}, 10^{-5} \mathrm{M}$ Pargyline, and $0.01 \%$ ascorbic acid (pH 7.5). Sections were then incubated for 60 minutes at room temperature in the same buffer with the addition of $0.9 \mathrm{nM}\left[{ }^{3} \mathrm{H}\right]-$ Rauwolscine, which binds (alpha) ${ }_{2}$-noradrenergic receptors. Nonspecific binding was determined on adjacent brain sections by adding both $0.9 \mathrm{nM}\left[{ }^{3} \mathrm{H}\right]$-Rauwolscine and $10 \mu \mathrm{M}$ Yohimbine to the buffer. Incubations were terminated by dipping slides in ice-cold buffer followed by two consecutive 5 minute washes in buffer. After the final wash, slides were rinsed in ice-cold distilled water and then dried at room temperature, at which point they were apposed to $\left[{ }^{3} \mathrm{H}\right]$-hyperfilm alongside microscale-calibrated tritium standards. After four months of apposition, films were developed and receptor binding was quantified using a computerized image analysis system (MCID-4, Imaging Research, St. Catherines, Ontario).

\section{Behavioral testing}

At approximately five months of age, animals from each group ( $n=16$ /group) were tested for immobility in the Porsolt forced swim test. In the Porsolt forced swim test, a rat is forced to swim in a confined space with no route of escape, and will cease to struggle and simply float after an initial period of vigorous activity (29). This behavior may be interpreted as a survival mechanism where the animal learns that struggling is hopeless and that immobility will minimize its energy expenditure, thus preventing drowning. Some investigators have labeled this as a test of behavioral despair because anti-depressants seem to increase the period spent struggling (30). This test has also been shown to be extremely sensitive to presynaptic (alpha) $)_{2}$ receptor agonists which inhibit NE release in the brain and subsequently increase time spent immobile (30).

Rats were injected either with the (alpha) $)_{2}$ receptor agonist, clonidine (100 micrograms $/ \mathrm{kg}$, i.p.), or an equivalent volume of saline. Twenty minutes after drug administration, animals were individually placed in a cylindrical Plexiglas container (height: $50 \mathrm{~cm}$, diameter: $30 \mathrm{~cm}$ ) filled to a depth of $40 \mathrm{~cm}$ with water maintained at room temperature. Rats were tested for 15 minutes and scored for time spent swimming. An animal was scored as being immobile when it ceased struggling or swimming and made minimal movements of its limbs to keep its head above water. 


\section{RESULTS}

\section{Receptor autoradiography}

The results from four brain sections analyzed from each birth condition group are summarized in Table 1.

A one-way analysis of variance (ANOVA) was used to determine differences in receptor binding between the groups in each brain region. Post hoc analysis was carried out using the Scheffe F-test. C-section with or without an additional period of anoxia induced greater receptor binding in the ventral hypothalamus (Figure 1) --an area including the arcuate nucleus, dorsomedial nucleus, and premammillary nucleus--when compared to vaginally-born controls $\left(F_{(2,11)}=26.27, p=0.0002\right)$. Animals born via C-section plus Anoxia showed less receptor binding in the $\mathrm{CA} 1$ region of the hippocampus when compared to Vaginal Controls or rats born via Caesarean alone $\left(F_{(2,11)}=42.77, p=0.0001\right)$. Conversely, the C-section plus Anoxia group exhibited greater receptor binding in the area of the posterior hypothalamus when compared to the two other groups $\left(F_{(2,11)}=\right.$ $9.422, p=0.0018)$. There were no observable changes between birth groups in the striatum, cortex, or dentate gyrus.

\section{Porsolt forced swim test}

A two-way ANOVA was used to determine differences between birth groups and drug treatment in time spent swimming (Figure 2). Swim time following clonidine treatment was significantly lower than that following saline treatment $\left(F_{(1,32)}=19.042, p=0.0001\right)$ for all birth groups. Hence, clonidine significantly increased immobility ( $p<0.05$; Tukey's HSD). There was a significant difference in time spent swimming between the Caesarian plus Anoxia group and the Vaginal Control group $\left(F_{(2,32)}=3.700, p=0.035\right)$ such that the Caesarean plus Anoxia group spent more time struggling when compared to aginally-born controls $(p<0.05$; Tukey's HSD). There was no significant interaction between drug treatment and birth group, i.e., the difference in time spent swimming between clonidine and saline treated animals was not dependent on birth group.

\section{DISCUSSION}

The current study demonstrated that a rat model of Caesarean-section birth, with or without an additional period of anoxia, produces an increase in (alpha) $)_{2}$ receptor density in the ventral hypothalamus of the young adult rat, suggesting that the Caesarean procedure itself is enough to induce long-lasting changes in noradrenergic systems in the brain. Interestingly, only the C-section plus Anoxia group showed a decrease in hippocampal, or increase in posterior hypothalamic, receptor binding. Thus, additional anoxia induces changes beyond the Caesarean effect. That $\mathrm{C}$-section alone induces long-term changes in catecholamine systems is in accordance with previous findings of the present authors and colleagues. It was found that the Caesarean procedure, with or without additional anoxia, was sufficient to facilitate stress-induced sensitization of nucleus accumbens dopamine release (26). Yet, it was also found that only the C-section plus anoxia birth condition sensitized animals to the locomotor stimulant effect of amphetamine (27). Thus, it appears that the Caesarean procedure used here is enough to evoke long-term changes in brain catecholamine systems, whereas the additional period of anoxia induces further qualitative alterations.

Most noradrenergic input to the cortex and associated structures is provided from cell bodies in the LC. These neurons play a role in orienting and attending to sudden contrasting or aversive stimulatory input (for review, see 31). Evidence suggests that NE increases the excitability of hippocampal neurons by acting on (beta)adrenergic postsynaptic receptors. Thus, the observed decrease in CA1 presynaptic (alpha) ${ }_{2}$-noradrenergic autoreceptor binding in anoxic animals (Caesarean plus Anoxia group) may serve to increase neuron excitability in this area by increasing NE release. 
The increase in (alpha) $)_{2}$ receptor binding seen in the ventral hypothalamus probably reflects an increase in either affinity or abundance of these receptors in the arcuate nucleus (see Figure 1). Most of the noradrenergic input to this area, as well as the posterior hypothalamus, derives from cell groups in the lower brainstem and LC (32). These NE afferents are thought to synapse onto DA cells in the arcuate that project in turn to the median eminence and play an inhibitory role in the release of prolactin as well as several hypothalamic releasing hormones (for review, see 33). Stressful stimuli induce prolactin release, probably via inhibition of hypothalamic DA neurons; although the precise mechanisms are not understood, these neurons may well be regulated by postsynaptic adrenergic receptors located on DA cells. Thus, because presynaptic (alpha) ${ }_{2}$ receptors reduce $\mathrm{NE}$ release, animals born via $\mathrm{C}$-section may possess an altered regulation of prolactin release (as well as hypothalamic releasing hormones) during stressful events. The role of prolactin during stress is undetermined; however, some evidence suggests that it may be involved in modulation of immunity by increasing antibody production and enhancing the lactogenic immune response in neonates (34). Accordingly, further investigation on anoxia at birth and its long-term effects on hypothalamic and pituitary hormones is merited.

In the present study, animals born via $\mathrm{C}$-section plus an additional period of anoxia showed reduced immobility in the forced swim test when compared to Vaginal Controls. While there was no significant statistical interaction, it can be seen in Figure 2 that the differences between the Vaginal Control group and Caesarean plus Anoxia group lie predominantly with those animals that received clonidine. Thus, the trend in these data indicate that $\mathrm{C}$-section plus Anoxia animals were less sensitive to the immobilizing effects of clonidine when compared to those born vaginally. Insofar as this is the case, it can be speculated that Csection plus Anoxia induces a long-term reduction in (alpha) $)_{2}$ receptor sensitivity to clonidine.

The overall significance of the present finding that $*_{2}$ receptor binding was increased in some brain regions while decreased in others is difficult to assess. Clonidine acts on (alpha) $)_{2}$ autoreceptors situated on NE neurons arising from the LC and brainstem regions, therefore these data suggest that the sum of (alpha $)_{2}$ receptors involved in the Porsolt forced swim task are either less sensitive or less abundant. Fewer or less sensitive (alpha) ${ }_{2}$-noradrenergic receptors would result in higher levels of NE release and subsequent increases in general anxiety and peripheral autonomic stress responses. Further research is needed to determine the role of the LC in these animals before any definitive conclusions may be drawn.

Factors intrinsic to the Caesarean procedure used here that may have contributed to the observed changes in (alpha) ${ }_{2}$ receptor binding and clonidine sensitivity have been discussed in detail elsewhere (26). Briefly, previous research has demonstrated that animals born by

C-section alone show reduced levels of plasma catecholamines at birth (35) and of plasma corticosteroids one hour after birth in comparison to vaginally born controls (Boksa, submitted). Since both catecholamines (36) and corticosteroids (37) promote maturation of lung function during the first few hours postnatally, these data suggest that delayed lung maturation is likely to occur following C-section birth, potentially resulting in transient hypoxia.

It should be noted that although rats born by $\mathrm{C}$-section with or without added anoxia show behavioral alterations on specialized testing, these animals appear normal on a gross behavioral level as adults and show no abnormalities on generalized testing of sensorimotor function (38). Rats born via C-section followed by 15 minutes of anoxia do, however, show subtle deficits in acquisition of a spatial learning task in the Morris water maze (38). Additionally, both animals born via C-section alone and those born by Caesarean with added anoxia exhibit reduced secretion of corticosterone in response to acute restraint stress, as well as reduced levels of corticosteroid type I receptors in hippocampus and hypothalamus (39). Such altered hormonal responses to stress may contribute to the altered behavioral responses associated with stress in these animals. 
The animal model of perinatal anoxia used here has several shortcomings if it is to be compared to the human condition. Rats are born at an earlier developmental stage than humans, such that the human brain at birth more resembles that of the 10 day old rat (40-42). Thus, this model may better reflect human prenatal anoxia if considered from a developmental standpoint. Furthermore, the current medical procedure of Caesarean delivery is much more sophisticated than that used for this animal model, with measures being taken to ensure that anoxia is avoided. However, studies have shown that despite these efforts, severe prolonged hypoxemia may occur in preterm human infants without changes in the rate of breathing or heart rate (43).

Taking into account the limitations of this model, it nonetheless has the potential to reveal how complications during the birthing event or early development may lead to long-term changes in brain development. As such, this model may be useful to study possible mechanisms underlying birth condition associated changes in brain development. Specifically, this is important for disorders that have been correlated with obstetric complications, such as schizophrenia.

In conclusion, C-section birth with an additional period of anoxia induces long-term increases in (alpha) ${ }_{2}^{-}$ noradrenergic receptor density in the hypothalamus while decreasing receptor density in the CA1 subfield of the hippocampus. Furthermore, this birth condition causes subsensitivity to clonidine in the forced swim test upon adulthood. These changes may be associated with a heightened sensitivity to stressful stimuli upon adulthood. This study underscores the need for further investigation into the NE stress responses in adult animals that received perinatal anoxia and the characterization of hypothalamic and pituitary hormones in these animals. Finally, considering the documented associations of schizophrenia with both obstetric complications and catecholamine dysfunction, the results of the current study are of considerable clinical interest and underscore the need for further research in this area.

\section{ACKNOWLEDGMENTS}

This research was supported by a grant from the Scottish Rite Schizophrenia Research Program, NMJ, USA and a postgraduate research scholarship awarded to W.G.B. from the Natural Sciences and Engineering Research Council (NSERC) of Canada. The support of Dr. Alain Gratton as well as the invaluable comments of Donna Toufexis are gratefully acknowledged.

\section{REFERENCES}

1. Brixey SN, Gallagher BJ, McFalls JA et al. Gestational and neonatal factors in the etiology of schizophrenia. Journal of Clinical Psychology 49(3): 447-456; 1993.

2. DeLisi LE, Dauphinais ID, and Gershon ES. Perinatal complications and reduced size of brain limbic structures in familial schizophrenia. Schizophrenia Bulletin 14(2): 185-191; 1988.

3. Günther-Genta F, Bovet P, Hohlfeld P. Obstetric complications and schizophrenia. A case-control study. British Journal of Psychiatry 164(2): 165-170; 1994.

4. Lewis SW, Murray RM. Obstetric complications, neurodevelopmental deviance, and risk of schizophrenia. Journal of Psychiatric Research 21(4): 413-421; 1987.

5. Norman RM, Malla AK. Stressful life events and schizophrenia I: a review of the research. British Journal of Psychiatry 162: 161-166; 1993.

6. O'Callaghan E, Gibson T, Colohan HA, et al. Risk of schizophrenia in adults born after obstetric complications and their association with early onset of illness: a controlled study. British Medical Journal 305(6864): 1256-1259; 1992. 
7. Pollack M, Woerner MG, Goodman W, et al. Childhood development patterns of hospitalized adult schizophrenic and non-schizophrenic psychiatric patients and their siblings. American Journal of Orthopsychiatry 36(3): 510-517; 1966.

8. Pollin W, Stabenau JR. Biological, psychological, and historical differences in a series of monozygotic twins discordant for schizophrenia. In: Rosenthal D, Kelly SS, eds. The Transmission of Schizophrenia. Oxford: Pergamon Press; 1968.

9. Verdoux H, Bourgeois M. A comparative study of obstetric history in schizophrenics, bipolar patients and normal subjects. Schizophrenia Research 9(1): 67-69; 1993.

10. Woerner MG, Pollack M, Klein DF. Pregnancy and birth complications in psychiatric patients: a comparison of schizophrenics and personality disorder patients with their siblings. Acta Psychiatrica Scandinavica 49(6): 712-721; 1973.

11. Connell PH. Amphetamine Psychosis. London: Chapman \& Hall; 1958.

12. Davis KL, Kahn RS, Ko G, et al. Dopamine in schizophrenia: a review and reconceptualization. American Journal of Psychiatry 148(11): 1474-1486; 1991.

13. Lieberman JA, Kinon BJ, Loebel AD. Dopaminergic mechanisms in idiopathic and drug-induced psychoses. Schizophrenia Bulletin, 16(1), 97-110; 1990.

14. Seeman P, Chau-Wong M, Tedesco J, et al. Brain receptors for antipsychotic drugs and dopamine: direct binding assays. Proceedings of the National Academy of Sciences, U.S.A. 72(11): 4376-4380; 1975.

15. Seeman P, Lee T, Chau-Wong M, et al. Antipsychotic drug doses and neuroleptic/dopamine receptors. Nature 261(5562): 717-719; 1976.

16. Creese I, Burt DR, Snyder SH. Dopamine receptor binding predicts clinical and pharmacological potencies of antischizophrenic drugs. Science 192(4238): 481-483; 1976.

17. Farley I, Price KS, Deck JH, et al. Norepinephrine in chronic paranoid schizophrenia: Above-normal levels in limbic forebrain. Science 200(4340): 456-458; 1978.

18. Farley IJ, Shannak KS, Hornykiewicz O. Brain monoamine changes in chronic paranoid schizophrenia and their possible relation to increased dopamine receptor sensitivity. Advances in Biochemical and Psychopharmacology 21: 427-433; 1980.

19. Gomes UC, Shanley BC, Potgieter L, et al. Noradrenergic overactivity in chronic schizophrenia: Evidence based on cerebrospinal fluid noradrenalin and cyclic nucleotide concentrations. British Journal of Psychiatry 137: 346-351; 1980.

20. Kemali D, Maj M, Galderisi S, et al. Factors associated with increased noradrenalin levels in schizophrenic patients. Progress in Neuro-Psychopharmacology and Biological Psychiatry 14(1): 49-59; 1990.

21. Kemali D, Vecchio MD, Maj M. Increased noradrenalin levels in CSF and plasma of schizophrenic patients. Biological Psychiatry 17(6): 711-717; 1982.

22. Lake CR, Sternberg DE, Van Kammen DP, et al. Schizophrenia: elevated cerebrospinal fluid norepinephrine. Science 207(4428): 331-333; 1980. 
23. Bjelke B, Andersson K, Ögren SO, et al. Asphytic lesion: proliferation of tyrosine hydroxylaseimmunoreactive nerve cell bodies in the rat substantia nigra and functional changes in dopamine neurotransmission. Brain Research 543(1): 1-9; 1991.

24. Loidl CF, Herrera-Marschitz M, Andersson K, et al. Long-term effects of perinatal asphyxia on basal ganglia neurotransmitter systems studied with microdialysis in rat. Neuroscience Letters 175(1-2): 9-12; 1994.

25. Dell'Anna E, Chen Y, Loidl F, et al. Short-term effects of perinatal asphyxia studied with Fosimmunocytochemistry and in vivo microdialysis in the rat. Experimental Neurology 131(2): 279-287; 1995.

26. Brake WG, Noel MB, Boksa P, et al. Influence of perinatal factors on the nucleus accumbens dopamine response to repeated stress during adulthood: an electrochemical study in the rat. Neuroscience 77: 10671076; 1997.

27. Brake WG, Boksa P, Gratton A. Anoxia at birth enhances sensitization to the locomotor stimulant effect of amphetamine in the adult rat. Psychopharmacology (In press).

28. Brady LS. Stress, antidepressant drugs, and the locus coeruleus. Brain Research Bulletin 35(5-6): 545$556 ; 1994$.

29. Porsolt RD, Anton G, Blavet N, et al. Behavioral despair in rats: a new model sensitive to antidepressant treatments. European Journal of Pharmacology 47(4): 379-391; 1978.

30. Parale MP, Kulkarmi SK. Clonidine - induced behavioral despair in mice: reversal by antidepressants. Psychopharmacology 89(2): 171-174; 1986.

31. Aston-Jones G, Foote SL, Bloom FE. Anatomy and physiology of the locus coeruleus neurons: Functional implications. In: Ziegler MG, Lake CR, eds. Norepinephrine. Frontiers of Clinical Neuroscience, Vol. 2. Baltimore: Williams \& Wilkins; 1984.

32. Zaborszky L. Afferent connections to the medial basal hypothalamus. Advances in Anatomy, Embryology and Cell Biology. 69: 1-107; 1982.

33. Everitt BJ, Meister B, Hökfelt T. The organization of monoaminergic neurons in the hypothalamus in relation to neuroendocrine integration. In: Nemeroff CB, ed. Neuroendocrinology. Boca Raton ; CRC Press; 1992.

34. Yirmiya R, Shavit Y, Ben-Eliyahu, S. et al. Modulation of immunity and neoplasia by neuropeptides released by stressors. In: McCubbin JA, Kaufmann PG, Nemeroff CB, eds. Stress, Neuropeptides, and Systemic Disease. San Diego: Academic Press; 1991.

35. El-Khodor BF, Boksa P. Long-term reciprocal changes in dopamine levels in prefrontal cortex versus nucleus accumbens in rats born by Caesarean section compared to vaginal birth. Experimental Neurology (In press).

36. Walters DV, Olver RE. The role of catecholamines in lung liquid absorption at birth. Pediatric Research 12: 239-242; 1978.

37. Fisher JH, McCormack F, Park SS, et al. In vivo regulation of surfactant proteins by glucocorticoids. American Journal of Respiratory Cell and Molecular Biology 5(1): 63-70; 1991. 
38. Boksa P, Krishnamurthy A, Brooks W. Effects of a period of asphyxia during birth on spatial learning in the rat. Pediatric Research 37(4 Pt 1): 489-496; 1995.

39. Boksa P, Krishnamurthy A, Sharma S. Hippocampal and hypothalamic type I corticosteroid receptor affinities are selectively reduced in adult rats born by a Caesarean procedure with or without an added period of anoxia. Neuroendocrinology 64(1): 25-34; 1996.

40. Dobbing J, Sands J. Comparative aspects of brain growth spurt. Early Human Development 3: 79-83; 1979.

41. Jilek L, Travnickova E, Trojan S. Characteristic metabolic and functional responses to oxygen deficiency in the central nervous system. In: Stave U, ed. Physiology of the Parinatal Period: Volume 2, Functional and Biochemical Development in Mammals. New York: Appleton-Century Crofts, Meredith Corporation; 1970.

42. Romijin HJ, Hofman MA, Gramsbergen A. At what age is developing rat cortex comparable to that of the full term human baby? Early Human Development 26: 61-67; 1991.

43. Poets CF, Stebbens VA, Richard D, et al. Prolonged episodes of hypoxemia in preterm infants undetectable by cardiorespiratory monitors. Pediatrics 95(6): 860-863; 1995.

\section{BIOGRAPHY}

Wayne Brake, B.Sc., M.Sc., received his B.Sc. in Psychology at Dalhousie University (Halifax, Nova Scotia, Canada) and his M.Sc. in Neuroscience at Carleton University (Ottawa, Ontario, Canada). In the autumn of 1997, he will have completed his Ph.D. in Neurology and Neurosurgery at McGill University (Montreal, Quebec, Canada) and will pursue a post-doctoral fellowship in neuroendocrinology at the Rockefeller University (New York, New York, U.S.A.).

Shakti Sharma, B.Sc., M.Sc., received his B.Sc. and M.Sc. degrees in biology and toxicology at Punjab University (Chandigarh, India) and is currently working in the Developmental Neuroendocrinology Lab at the Douglas Hospital Research Centre (Montreal, Quebec, Canada).

Copyright (C) 1997 by MJM 